\title{
Influence of pitch size and goalkeepers on external and internal load during small-sided games in amateur soccer players
}

\author{
Roberto Modena $^{1,2}(\mathbb{D}) \cdot$ Andrea Togni $^{1} \cdot$ Maurizio Fanchini ${ }^{1,3}(\mathbb{D}) \cdot$ Barbara Pellegrini $^{1,2}$ (D) $\cdot$ Federico Schena ${ }^{1,2}$ (D)
}

Received: 2 February 2021 / Accepted: 17 April 2021 / Published online: 3 May 2021

(c) The Author(s) 2021

\begin{abstract}
Purpose To analyse the influence of goalkeepers during 4-a-side small-sided games, played in pitches of two different sizes (small: $30 \times 20 \mathrm{~m}$, large: $40 \times 30 \mathrm{~m}$ ).

Methods Total distance covered (TD), distance covered at low- (LSD), moderate- (MSD), high- (HSD) and very high-speed (VHSD), average and maximal speed, Edwards' training load (Edw-TL), time spent above 90\% of maximal heart rate (T90\%) and rate of perceived exertion (RPE) were monitored, in 18 amateur soccer players.

Results Higher TD (mean difference: $+181 \mathrm{~m}$, Hedge's $g: 0.93$ and $+400 \mathrm{~m}, 3.37)$, MSD (+ $85 \mathrm{~m}, 0.79$ and $+146 \mathrm{~m}$, 1.64), HSD (+101 m, 1.41 and $+179 \mathrm{~m}, 3.26)$, VHSD (+30 m, 1.89 and $+35 \mathrm{~m}, 1.26)$, average speed $\left(+0.65 \mathrm{~km} \mathrm{~h}{ }^{-1}, 0.88\right.$ and $\left.+1.47 \mathrm{~km} \mathrm{~h}^{-1}, 3.31\right)$ and maximal speed $\left(+3.60 \mathrm{~km} \mathrm{~h}^{-1}, 1.40\right.$ and $\left.+3.58 \mathrm{~km} \mathrm{~h}^{-1}, 1.40\right)$ were found in large than small pitch, without and with goalkeepers, respectively. Goalkeeper's presence increased Edw-TL (+8.4 AU, 0.70) and reduced TD $(-141 \mathrm{~m}, 0.75)$, HSD $(-54 \mathrm{~m}, 0.75)$ and average speed $\left(-0.54 \mathrm{~km} \mathrm{~h}^{-1}, 0.76\right)$ in small pitch and maximal speed $(1.59 \mathrm{~km}$ $\mathrm{h}^{-1}, 0.60$ and $\left.1.61 \mathrm{~km} \mathrm{~h}^{-1}, 0.66\right)$ in both small and large pitches, respectively. RPE was higher $(+20,1.52)$ in the large than small pitch when the goalkeepers were present.

Conclusion Implementing small-sided games, coaches should be aware that lower external load with similar internal load could be provided using small pitch with goalkeeper rather than either small goals or larger pitch. Furthermore, large smallsided games without goalkeeper may be the best choice for eliciting high training load.
\end{abstract}

Keywords Football $\cdot$ SSGs $\cdot$ Pitch dimensions $\cdot$ Small goals $\cdot$ Heart rate $\cdot$ RPE

$\begin{array}{ll}\text { Abbreviations } \\ \text { SSGs } & \text { Small-sided games } \\ \text { RPE } & \text { Rate of perceived exertion } \\ \text { GPS } & \text { Global positioning system } \\ \text { TD } & \text { Total distance } \\ \text { LSD } & \text { Low-speed distance } \\ \text { MSD } & \text { Moderate-speed distance } \\ \text { HSD } & \text { High-speed distance } \\ \text { VHSD } & \text { Very high-speed distance }\end{array}$

Roberto Modena

roberto.modena@univr.it

1 Department of Neurosciences, Biomedicine and Movement Sciences, University of Verona, Verona, Italy

2 CeRiSM, Sport, Mountain and Health Research Centre, University of Verona, via Matteo del Ben 5/b, 38068 Rovereto, Italy

3 AS Roma Performance Department, AS Roma Football Club, Roma, Italy
Edw-TL Edwards' training load

T90\% Time spent above $90 \%$ of maximal heart rate

\section{Introduction}

Performance has been suggested as a multifactorial construct in the theoretical simplified model of football (soccer) performance [1] and its improvement is currently one of the main purposes of the training process [2]. From a holistic and time-efficient perspective, particularly important at amateur level (given the low number of weekly training sessions), coaches often use an integrated approach, for example using small-sided games (SSGs), to provide a specific training stimuli and simultaneously improve both technicaltactical and physical characteristics of the players [3, 4]. The training load stimuli provided by SSGs can be managed by modifying several variables such as pitch' size $[5,6]$, number of players involved [5, 7, 8], rules [9], floater's use $[10,11]$, duration and numbers of bouts $[12,13]$ and the 
presence of goalkeepers [7, 8, 14-16]. Even if the available evidence is scarce, the presence of goalkeepers and regular goals, compared to possession format and use of small goals, may be important not only to develop tactical and technical skills (i.e., kick), but also to increase the active participation of the goalkeeper when the team is in ball-possession [17]. At present, most of the studies examined separately the effect of area per player (ratio between pitch's size and number of players) and the presence of goalkeeper, but few of them considered their interaction [18].

Studies that examined the effect of area per player on heart rate responses provide contradictory results. For example, Hodgson et al. [19] and Kelly and Drust [20] found no effect on heart rate when the area per player was increased while greater responses were found in other studies $[5,16$, $21,22]$. Studies that evaluated the rate of perceived exertion (RPE), showed higher rating values when the area per player increased [5, 16, 21, 22]. Furthermore, wider area per player seems to lead an increase in time-motion metrics (i.e., total distance covered, distance covered at high speed and accelerations) $[6,8,19,21]$.

Regarding the effect of the presence of goalkeepers in SSGs, the heterogeneity of formats and rules used in the studies limits the comparisons. However, it seems that the presence of goalkeepers tends to decrease both heart rate response and physical performance $[6-8,11,15,16]$. In particular, Gaudino et al. [8] showed an increase of high-speed distance and maximal speed whereas Castellano et al. [7] and Koklu et al. [15] reported a decrement of player load and high-speed distance, respectively.
Furthermore, studies that examined the effect of the goalkeeper's presence in SSGs have involved young soccer players $[11,15,16]$ or adult high-level players (semi-professional and professional) $[7,8]$, therefore information on amateur soccer is needed.

The aim of this study was to examine the effect of the interaction between the goalkeeper's presence and two different pitch dimensions, during a 4-a-side SSGs, on the external and internal load values in adult amateur soccer players. Time motion parameters, Edward's training load, time spent above $90 \%$ of maximal heart rate and rate of perceived exertion were considered as outcomes. Based on the present knowledge, we expected that the presence of goalkeepers may reduce physiological responses and time motion parameters, especially in the small pitch. Moreover, we hypothesized that the gap in the external and internal load, measured during SSGs played in the large than in small pitch (higher in the large one), can be lowered by the presence of goalkeepers.

\section{Methods}

\section{Experimental protocol}

In this observational study, data were collected throughout the in-season period (season 2017/2018) during the usual training sessions of an amateur soccer team. Each SSGs session was performed once a week, at least $48 \mathrm{~h}$ after the last match and after a standardized warm-up to limit the

Table 1 Interaction between pitch size and goalkeepers' presence

\begin{tabular}{|c|c|c|c|c|c|}
\hline & \multicolumn{2}{|l|}{ Small pitch $(30 \times 20 \mathrm{~m})$} & \multicolumn{2}{|l|}{ Large pitch $(40 \times 30 \mathrm{~m})$} & \multirow{2}{*}{$\begin{array}{l}\text { Interaction pitch } \\
\text { size } \times \text { GK pres- } \\
\text { ence } \\
p \text { value }\end{array}$} \\
\hline & Without goalkeepers & With goalkeepers & Without goalkeepers & With goalkeepers & \\
\hline $\mathrm{TD}[\mathrm{m}]$ & $\begin{array}{l}1620.11[1542.78- \\
1701.32]\end{array}$ & $\begin{array}{l}1478.79[1424.96- \\
1534.67]\end{array}$ & $\begin{array}{l}1801.45[1733.16- \\
1872.43]\end{array}$ & $\begin{array}{l}1878.52[1825.84- \\
1932.73]\end{array}$ & $<0.001$ \\
\hline $\operatorname{LSD}[\mathrm{m}]$ & $\begin{array}{l}1223.77[1203.94- \\
1243.92]\end{array}$ & $\begin{array}{l}1170.55 \text { [1114.88- } \\
1229]\end{array}$ & $\begin{array}{l}1184.32[1148.53- \\
1221.21]\end{array}$ & $\begin{array}{l}1207.45[1175.5- \\
1240.26]\end{array}$ & 0.005 \\
\hline $\operatorname{MSD}[\mathrm{m}]$ & $264.56[220.88-316.86]$ & 234.47 [195.7-280.93] & 349.49 [326.36-374.26] & 380.51 [343.29-421.76] & $<0.001$ \\
\hline HSD [m] & 123.46 [95.94-158.87] & 69.46 [53.4-90.34] & 224.93 [206.97-244.46] & $248.62[221.29-279.31]$ & $<0.001$ \\
\hline VHSD [m] & $12.27[8.61-17.49]$ & 6.89 [4.34-10.92] & $42.71[34.12-53.45]$ & 41.94 [28.39-61.97] & $<0.001$ \\
\hline AvgSpeed $\left[\mathrm{km} \mathrm{h}^{-1}\right]$ & $6.07[5.77-6.38]$ & $5.52[5.32-5.73]$ & $6.72[6.47-6.99]$ & $6.9[6.79-7.19]$ & $<0.001$ \\
\hline MaxSpeed $\left[\mathrm{km} \mathrm{h}^{-1}\right]$ & 20.63 [19.65-21.67] & $19.04[17.92-20.23]$ & $24.24[23.25-25.26]$ & $22.62[21.48-23.83]$ & $<0.001$ \\
\hline $\mathrm{T} 90 \%[\mathrm{~min}]$ & $0.42[0.28-0.64]$ & $0.57[0.47-0.7]$ & $0.52[0.39-0.69]$ & $0.56[0.43-0.73]$ & 0.272 \\
\hline Edw-TL [AU] & $60.24[55.2-65.74]$ & $68.62[66.08-71.26]$ & $62.43[57.3-68.02]$ & $66.76[63.43-70.26]$ & 0.011 \\
\hline RPE [AU] & 53.18 [46.35-61.02] & 48.19 [42.16-55.07] & 56.83 [49.17-65.69] & 68.47 [62.89-74.55] & $<0.001$ \\
\hline
\end{tabular}

Estimated marginal means [95\% CI] and $p$ value for pitch' size and goalkeepers interaction effect of time-motion characteristics, heart rate responses and RPE during four different small-sided game's formats

$T D$ total distance, $L S D$ low speed distance, $M S D$ moderate speed distance, $H S D$ high speed distance, VHSD very high speed distance, AvgSpeed average speed, MaxSpeed maximal speed, T90\% time spent above $90 \%$ of maximal heart rate, Edw-TL Edward's training load, $R P E$ rate of perceived exertion 


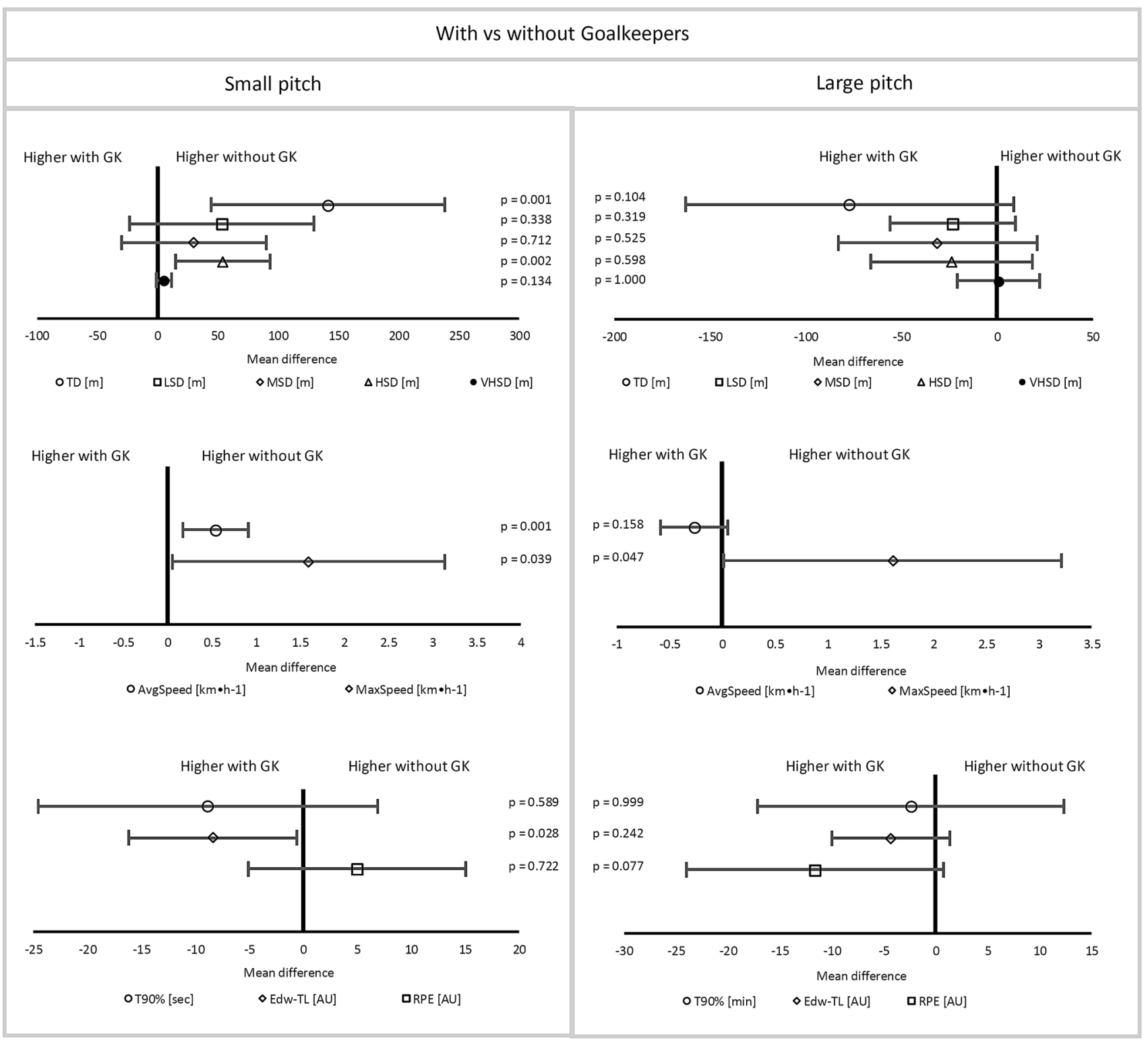

Fig. 1 Comparison between with and without goalkeepers. Mean differences with $95 \%$ CI for pairwise comparison between SSGs with and without goalkeepers; $30 \times 20 \mathrm{~m}$ (left) and $40 \times 30 \mathrm{~m}$ (right). $G K$ goalkeepers, $T D$ total distance, $L S D$ low speed distance, $M S D$ moder-

potential confounder effect of the activity carried out before the examined SSGs (e.g., post-activation potentiation and/or fatigue). A $2 \times 2$ design was used, where the two independent factors were the pitch size (small and large) and the presence of goalkeepers (goalkeepers with regular goals and no goalkeepers with small goals). Load markers were collected for every SSGs using Global Positioning System (GPS) for external load, heart rate and rate of perceived exertion (RPE) for internal load. Before data collection all the participants ate speed distance, $H S D$ high speed distance, $V H S D$ very high speed distance, AvgSpeed average speed, MaxSpeed maximal speed, T90\% time spent above $90 \%$ of maximal heart rate, EdwTL Edwards' training load, $R P E$ rate of perceived exertion

performed 2 weeks of familiarization with the RPE method using the CR100 Borg scale (CR100). Yo-Yo Intermittent Recovery Test level 1 was used to anchoring the player's RPE (i.e., CR100) to exercise's intensity [23] as well as to assess each player's level of the endurance physical performance and maximal heart rate. A standardized 18-min warm-up based on jogging, dynamic stretching, sprinting, acceleration and deceleration drills was completed before the Yo-Yo Intermittent Recovery Test and each SSGs session. 


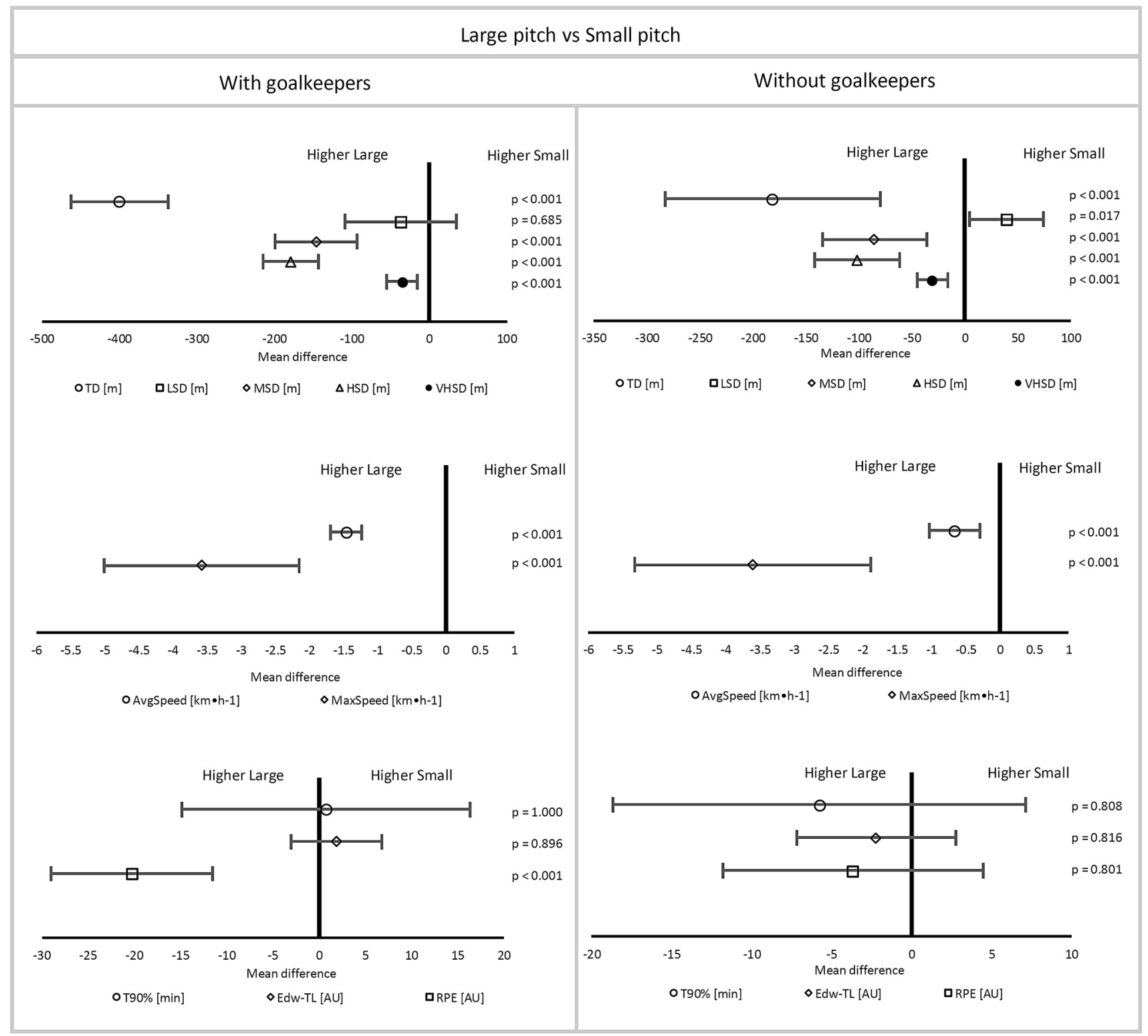

Fig. 2 Comparisons between large and small pitch. Mean differences with $95 \% \mathrm{CI}$ for pairwise comparison between large $(40 \times 30 \mathrm{~m})$ and small $(30 \times 20 \mathrm{~m})$ pitch SSGs; with goalkeepers (left) and without goalkeepers (right). $T D$ total distance, $L S D$ low speed distance, $M S D$

\section{Population}

Eighteen amateur male soccer players took part in the study. All the participants had at least 8 years of regular experience in an organized soccer environment (training days $\geq 2$ per week; official competition $\geq 1$ per week). At the end of data collection, only 13 players (age $28.7 \pm 6.3$ years, body mass $73.8 \pm 6.1 \mathrm{~kg}$, height $178.0 \pm 0.1 \mathrm{~cm}$ ) had at least 1 valid measurement for each condition and could be included in the final analysis. moderate speed distance, $H S D$ high speed distance, $V H S D$ very high speed distance, AvgSpeed average speed, MaxSpeed maximal speed, T90\% time spent above $90 \%$ of maximal heart rate, EdwTL Edward's training load, $R P E$ rate of perceived exertion

\section{Small-sided games}

SSGs were performed on an artificial 3G rubber crumb turf as 4-a-side matches consisting of four bouts of 4 min separated by $3 \mathrm{~min}$ of passive recovery. Four randomly ordered type of SSGs $(n=81)$ were performed with and without goalkeepers and considering small $(30 \times 20 \mathrm{~m})$ and large $(40 \times 30 \mathrm{~m})$ pitch dimension (area per player of 75 and 150 $\mathrm{m}^{2}$ without goalkeepers, respectively) [5]. During the SSGs without goalkeepers, players had to score in a small goal 
$(2 \times 1 \mathrm{~m})$, whereas when goalkeepers were present players had to score in a regular goal. Official rules of 11-a-side football was used except for offside rule. To avoid roster's imbalance, the composition of the teams was based on playing positions and technical abilities (assessed by coach). In addition, to provide high motivation, SSGs have been played in a tournament scenario, with points awarded for winning ( +3 points), drawing ( +1 point) and goal scoring $(+1$ point). Balls were placed around the perimeter of the pitch and quickly returned when went out of play, avoiding long interruptions of activity. Furthermore, to ensure high intensity effort, a verbal encouragement was provided by the head coach of the team [5].

\section{External load}

Outfield player's external load was monitored during the SSGs by a 10-Hz-GPS device (Viper, StatSports, Ireland). Previous studies demonstrated a good accuracy of this device to measure distance and peak velocity [24]. Players wore a tight-fitting vest with the GPS units placed into a pocket between shoulder's blades. Every GPS unit was turned on and placed outdoors 15 min prior the start of the session to ensure a better satellite's signals reception [25]. The following time motion characteristics were chosen as representative of external training load: total distance covered (TD), distance covered at different speed zones (lowspeed distance [0-10.8 $\mathrm{km} \mathrm{h}^{-1}$, LSD], moderate-speed distance [10.8-14.4 $\mathrm{km} \mathrm{h}^{-1}$, MSD], high-speed distance [14.4-19.8 $\mathrm{km} \mathrm{h}^{-1}$, HSD] and very high-speed distance $\left.\left[\geq 19.8 \mathrm{~km} \mathrm{~h}^{-1}, \mathrm{VHSD}\right]\right)$, average speed and maximal speed [26].

\section{Internal load}

Internal load was assessed with heart rate and RPE. Heart rate was monitored at 1-s intervals using a heart rate monitor band (Polar ${ }^{\circledR}$ T31) connected with the GPS device which stored data synchronized with player's movements. For every session, time spent in five intensity zones, based on a percentage of maximal heart rate (zone 1: 50-60\%, zone $2: 60-70 \%$, zone $3: 70-80 \%$, zone $4: 80-90 \%$, and zone 5: $90-100 \%$ ), was quantified and multiplied for a coefficient, different for each zone (from 1 to 5 related to the intensity); the sum of the five products obtained was used as training load based on heart rate as proposed by Edwards (Edw-TL) [27] and used in several previous studies on football $[28,29]$. Furthermore, also time spent above $90 \%$ of maximal heart rate (T90\%) was considered as internal load parameter and a relevant factor to improve aerobic fitness [30]. In addition, players were asked, immediately at the end of the SSGs, to provide RPE rating in a confidential way, to avoid influence of teammates. The CR100-Borg scale was used, as it was shown to be valid in soccer context [29, 31], in addition participants performed both memory and exercise anchoring before the commencement of data collection.

\section{Statistical analysis}

Normal distribution of the data was checked using Shapiro-Wilk test with the level of statistical significance set at 0.05 . Pitch' size and goalkeepers' presence were used as independent variables whereas GPS metrics, heart rate parameters and RPE were considered as dependent variables. Since the different number of participants' repeated measures, generalized estimating equations (GEE) analysis, with exchangeable correlation structure, was used to examine interaction between the two independent variables. GEE Log distribution was selected when variables were not normally distributed, otherwise linear distribution was chosen. When a significant interaction effect was found $(p<0.05)$, estimated marginal means pairwise comparisons with Sidak adjustment were performed for investigating significant differences and Hedges' $g$ was used as standardized effect size [32]. Estimated means and mean's differences for pairwise comparisons are presented with $95 \%$ confidence interval $(95 \% \mathrm{CI})$. All the statistical analyses were performed with SPSS software (IBM SPSS Statistics, version 22.0).

\section{Results}

External and internal loads estimated means with 95\% CI and $p$ values of interactions between pitch' size and goalkeeper's presence are presented in Table 1, as well as mean difference with 95\% CI for pairwise comparisons are presented in Figs. 1 and 2. Hedges' $g$ is presented in the text as a measure of standardized effect size.

The distance covered during Yo-Yo intermittent recovery test level 1 was $1766 \pm 304 \mathrm{~m}$ and maximal heart rate was $181 \pm 10 \mathrm{bpm}$.

\section{Pitch size effect}

A significant effect of the size of the pitch was found, with higher values in large compared to small pitch, for TD (+291.7 m [244.5-339.0], $p<0.001, g=1.59$ [1.09-2.10]), MSD (+ 115.6 m [88.6-142.6], $p<0.001, g=1.03$ [0.57-1.50]), HSD (+143.9 m [125.3-162.4], $p<0.001$. $g=2.27$ [1.71-2.84]), VHSD (+33.1 m [24.0-42.3], $p<0.001, g=1.38$ [0.90-1.87]), average speed $\left(+1.1 \mathrm{~km} \mathrm{~h}^{-1}\right.$ [0.9-1.2], $p<0.001, g=1.55$ [1.05-2.05]), maximal speed 
$\left(+3.6 \mathrm{~km} \mathrm{~h}^{-1}[2.6-4.6], p<0.001, g=1.18[0.71-1.66]\right)$ and RPE (+ 11.8 [6.1-17.4], $p<0.001, g=0.63$ [0.18-1.08]).

\section{Goalkeepers' presence effect}

A main effect of goalkeepers' presence was found on some assessed parameters, in fact, HSD ( $-35.2 \mathrm{~m}[-59.9$ to $-10.6], p=0.005, g=0.45$ [0.01-0.89]), VHSD $(-5.9 \mathrm{~m}$ [ -11.2 to -0.6$], p=0.029, g=0.33$ [ -0.11 to 0.77$])$ and maximal speed $\left(-1.6 \mathrm{~km} \mathrm{~h}^{-1}[-2.6\right.$ to -0.6$], p=0.002$, $g=0.55[0.10-1.00])$ were lower while Edw-TL $(+6.4$ [2.2-10.5], $p=0.003, g=0.45[0.01-0.90])$ was higher when the goalkeepers were present compared to small goals format.

\section{Goalkeepers' presence and pitch' size interaction}

A significant interaction between pitch' size and goalkeepers' presence was found for TD, MSD, HSD, VHSD, average speed, maximal speed, RPE (all $p<0.001), \operatorname{LSD}(p=0.005)$ and Edw-TL $(p=0.011)$ (Table 1). The presence of goalkeepers reduced TD $(g=0.75[0.11-1.38])$, HSD $(g=0.75$ [0.11-1.38]), average speed $(g=0.76[0.12-1.39])$, maximal speed ( $g=0.60[-0.03$ to 1.22$])$ and RPE ( $g=0.28[-0.34$ to 0.90$]$ ) in the small pitch (Fig. 1) whilst lowered only the maximal speed ( $g=0.66[-0.01$ to 1.32$])$ in the large pitch (Fig. 1).

The use of a larger pitch led to an increase in TD $(g=3.37[2.32-4.44]$ and $g=0.93[0.31-1.56])$, MSD $(g=1.64[0.87-2.43]$ and $g=0.79[0.18-1.41])$, HSD $(g=3.26[2.23-4.31]$ and $g=1.41[0.75-2.08])$, VHSD $(g=1.26[0.53-2.00]$ and $g=1.89[1.18-2.61])$, average speed $(g=3.31$ [2.27-4.37] and $g=0.88$ [0.26-1.51]) and maximal speed ( $g=1.40$ [0.66-2.16] and 1.4 [0.74-2.07]) both in presence and absence of goalkeepers, respectively (Fig. 2). Furthermore, RPE was higher in the large than small pitch only when the goalkeepers were present $(g=1.52$ [0.77-2.29]) (Fig. 2). On the contrary, the goalkeepers' participation increased Edw-TL only when the small pitch was used $(g=0.70[0.07-1.34])$.

\section{Discussion}

The results of the present study showed higher external training load and RPE in the large than small pitch. The goalkeepers' presence decreased running distances at highintensity speed and increased the EdwTL when compared with small goals. Furthermore, we found that the effect of the goalkeepers' presence on external and internal load metrics values (except for T90\%), is dependent by the pitch size.

\section{Pitch'size effect}

Our results, showed higher RPE $(g=0.63)$ and no changes in heart rate responses in large compared to small pitch. Rampinini et al. [5] found an increase on both RPE and heart rate in the large than both medium and small pitches. Moreover, the increased external load in the large pitch showed by our results (i.e., TD: $g=1.59$; MSD: $g=1.03$; HSD: $g=2.27$; VHSD: $g=1.38$; average speed: $g=1.55$; maximal speed: $g=1.18$ ) confirmed those of previous studies [6, 8, 19, 21]. For example, Casamichana et al. [21] compared three pitches of different size, finding that external load metrics increased as the pitch size became wider, especially comparing large to small pitches. Recently, Riboli et al. [6] showed strong positive relationships between the area per player and several motion metrics (e.g., total distance and high-intensity distance) in smallsided games with several formats.

\section{Goalkeepers' presence effect}

Our findings, showing that the goalkeepers' presence lowered high-intensity metrics (i.e., HSD: $g=0.45$; VHSD: $g=0.33$; maximal speed: $g=0.55$ ), confirmed those reported by previous studies, with the exception of Gaudino et al. [8] who found higher values of high-speed distance and maximal speed. On the other hand, in contrast with other studies, we found higher heart rate responses (i.e., EdwTL: $g=0.45$ ) when goalkeepers were present.

\section{Pitch' size and goalkeepers' presence interaction}

An interaction effect between pitch' size and goalkeepers' presence could represent one of the reasons of inconsistent results among studies that examined the effect of goalkeepers' presence. Our results showed that Edw-TL was increased $(g=0.70)$ due to the presence of goalkeepers, only in the small pitch; this effect may be due to the presence of a fifth player who can probably elevate the pace of the play and the technical action rate which may have an impact on the heart rate response. This difference was not significant in the large pitch, probably because the impact of fifth player in a wider area is not sufficient to increase Edw-TL. These findings are in contrast with Hulka et al. [16] who found higher average heart rate and time spent between 65 and 85\% of maximum heart rate in small pitch 5-a-side SSGs played without goalkeepers.

Regarding the rate of perceived exertion, Hulka et al. [16] showed no differences in RPE between format with and without goalkeepers while Koklu et al. [15] found higher RPE in SSGs with goalkeepers and a low number 
of player (but the same area per player). Our data partially agreed with previous findings, showing that the presence of goalkeepers seems not to influence RPE in large pitches but may affect it in small pitches (i.e., lower in small pitch with goalkeepers compared to small goals, $g=0.28$ ). Moreover, RPE was increased going from the small to large pitch with goalkeepers $(g=1.52)$ but no differences exist between the two dimensions without goalkeepers. Probably, the reduction in external load, provided by adding a player to each team, caused a greater reduction in RPE in the small compared to large pitch. Interesting, similar RPE (53 and 57) between small and large pitches without goalkeepers corresponded with higher external load (i.e., TD: $g=0.93$; MSD: $g=0.79$; HSD: $g=1.41$; VHSD: $g=1.89$; average speed: $g=0.88$; maximal speed: $g=1.4$ ) when the playing area was wider. Together, these results suggested that the absence of goalkeepers allows greater external load responses in the larger pitch without increasing perception of effort. The increase in the external load may have had an impact on muscular rather than overall perception, thus it may be reasonable for both coaching practice and future research using differential RPE [33] to assess SSGs training load.

The analysis of interaction between pitch' size and goalkeepers' presence highlighted that the presence of goalkeepers seems to influence time-motion characteristics only when SSGs were played in the small pitch. TD $(g=0.75)$, HSD $(g=0.75)$, average speed $(g=0.76)$, maximal speed $(\mathrm{g}=0.60)$ were greater in the small pitch without goalkeepers compared to the same pitch with goalkeepers, showing that adding the goalkeepers when the available playing surface is already small can result in a decrease of intensity and amount of motion. The presence of two additional players (two goalkeepers) had probably a greater relative weight in the small pitch than in the large one. Similar results were found by Gaudino et al. [8] and Riboli et al. [6], however, a comparison can be flawed because in their study the use of several formats and rules may have influenced the effect of interaction between the presence of goalkeepers and the dimension of the pitch. On the contrary, Hulka et al. [16] found that the distance covered was higher with goalkeepers than without in small pitch and no differences were found in medium and large pitches. Differences between our study and that of Hulka et al. may be explained by the dimension of the pitch used, $75 \mathrm{~m}^{2}$ and $60 \mathrm{~m}^{2}$ per player in the present study (without and with goalkeepers, respectively) that was similar to the medium size pitch of Hulka et al. (i.e., 56 $\mathrm{m}^{2}$ and $47 \mathrm{~m}^{2}$ in the small pitch, $87.5 \mathrm{~m}^{2}$ and $73 \mathrm{~m}^{2}$ in the medium pitch, and $126 \mathrm{~m}^{2}$ and $105 \mathrm{~m}^{2}$ in the large pitch, without and with goalkeepers, respectively). [16] Furthermore, in contrast to Gaudino et al.[8] who showed higher maximal speed in SSGs with goalkeepers than possession format, we found higher maximal speed when the goalkeepers were not present, in both small $(g=0.60)$ and large pitch
( $g=0.66)$. It may occur because players have to quickly manage transition phases to cover the own goal or score a goal taking advantage of imperfect positioning of opponents.

Our results can provide practical useful information for coaches of amateur teams, training ideally three times a week and compete once a week. As found also in previous studies $[6,8,21]$, a coach could plan a small-sided game in a small pitch to provide a low mechanical work (i.e., during a return to play session or a light session throughout the inseason period). Based on our results, the external load may be further lowered using the goalkeepers rather than small goals. Furthermore, when coaches need to provide a high external load, they should consider that the large pitch without goalkeepers allows to elicit greater external load than small pitch but with similar overall perceived of exertion. A practical example is the first training session after the match (i.e., $M D+2$ ), when starters (the players who played from the start the previous match) should participate in SSGs with small area per player and the presence of goalkeeper whereas no-starters (the players who did not play or enters during the game) should be involved in SSGs played in a wider pitch without goalkeeper. Moreover, the session training in the middle of the week, typically aims to develop speed and power capacity that may be stimulated better by a pitch of large dimension with small goals than with regular goals and goalkeepers, allowing to reach higher maximal speed values.

The present study is not free of some limitations. Smallsided games were performed $48 \mathrm{~h}$ after the match, however not every player was equally involved in the match, therefore individual recovery conditions might have influenced the outcomes. Moreover, since the SSGs were monitored throughout several weeks, the cumulated training load and fatigue of each participant might have been different throughout the sessions. Verbal encouragement and a tournament scenario were proposed to increase the players' motivation, but the efficacy of these intervention on each player might not have been the same, having different impact on the parameters assessed. External load metrics assessed with GPS (e.g., high- and very-high intensity running distances) need to be deeper verified in their validity and reliability during small sided games, and this may have influenced the results. In addition, the conclusions of the present study may be flawed by the small number of participants and the interindividual variability. Further investigations needed to better elucidate these phenomena at amateur level.

\section{Conclusions}

Our findings confirmed the literature in fact, 4-a-side smallsided games played in higher area elicited greater training load. Moreover, our results added information about amateur soccer players, showing that the effect of the presence 
of goalkeepers is dependent from playing area and is more relevant on time motion characteristics than heart rate and perceived responses. The players reached higher amount of total distance and high-speed distance, with lower heart rate responses, with the use of small goals instead of regular goals and goalkeepers when the area per player was small.

Acknowledgments We would like to thank Prof. Leonardo Tartaruga for his precious help in GEE approach for statistical analysis.

Funding Open access funding provided by Università degli Studi di Verona within the CRUI-CARE Agreement.

\section{Declarations}

Conflict of interest All of authors declare to have no conflict of interest.

Ethical statement The study was conducted according to the Declaration of Helsinki and written informed consent was obtained from the subjects before the commencement of the study.

Open Access This article is licensed under a Creative Commons Attribution 4.0 International License, which permits use, sharing, adaptation, distribution and reproduction in any medium or format, as long as you give appropriate credit to the original author(s) and the source, provide a link to the Creative Commons licence, and indicate if changes were made. The images or other third party material in this article are included in the article's Creative Commons licence, unless indicated otherwise in a credit line to the material. If material is not included in the article's Creative Commons licence and your intended use is not permitted by statutory regulation or exceeds the permitted use, you will need to obtain permission directly from the copyright holder. To view a copy of this licence, visit http://creativecommons.org/licenses/by/4.0/.

\section{References}

1. Impellizzeri FM, Marcora SM (2009) Test validation in sport physiology: lessons learned from clinimetrics. Int J Sports Physiol Perform 4(2):269-277. https://doi.org/10.1123/ijspp.4.2.269

2. Stølen T, Chamari K, Castagna C, Wisløff U (2005) Physiology of soccer: an update. Sports Med 35(6):501-536. https://doi.org/ 10.2165/00007256-200535060-00004

3. Brandes M, Elvers S (2017) Elite youth soccer players' physiological responses, time-motion characteristics, and game performance in 4 vs. 4 small-sided games: The influence of coach feedback. J Strength Cond Res 31(10):2652-2658. https://doi.org/10.1519/ JSC.0000000000001717

4. Davids K, Araújo D, Correia V, Vilar L (2013) How small-sided and conditioned games enhance acquisition of movement and decision-making skills. Exerc Sport Sci Rev 41(3):154-161. https://doi.org/10.1097/JES.0b013e318292f3ec

5. Rampinini E, Impellizzeri FM, Castagna $C$ et al (2007) Factors influencing physiological responses to small-sided soccer games. J Sports Sci 25(6):659-666. https://doi.org/10.1080/0264041060 0811858

6. Riboli A, Coratella G, Rampichini S, Ce E, Esposito F (2020) Area per player in small-sided games to replicate the external load and estimated physiological match demands in elite soccer players. PLoS ONE. https://doi.org/10.1371/journal.pone.02291 94

7. Castellano J, Casamichana D, Dellal A (2013) Influence of game format and number of players on heart rate responses and physical demands in small-sided soccer games. J Strength Cond Res 27(5):1295-1303. https://doi.org/10.1519/JSC.0b013e3182 $67 \mathrm{a} 5 \mathrm{~d} 1$

8. Gaudino P, Alberti G, Iaia FM (2014) Estimated metabolic and mechanical demands during different small-sided games in elite soccer players. Hum Mov Sci 36:123-133. https://doi.org/10. 1016/j.humov.2014.05.006

9. Hill-Haas SV, Coutts AJ, Dawson BT, Rowsell GJ (2010) Timemotion characteristics and physiological responses of small-sided games in elite youth players: the influence of player number and rule changes. J Strength Cond Res 24(8):2149-2156. https://doi. org/10.1519/JSC.0b013e3181af5265

10. Lacome M, Simpson BM, Cholley Y, Buchheit M (2018) Locomotor and heart rate responses of floaters during small-sided games in elite soccer players: effect of pitch size and inclusion of goalkeepers. Int J Sports Physiol Perform. https://doi.org/10. 1123/ijspp.2017-0340

11. Mallo J, Navarro E (2008) Physical load imposed on soccer players during small-sided training games. J Sports Med Phys Fitness 48(2): 166-171

12. Fanchini M, Azzalin A, Castagna C, Schena F, Mccall A, Impellizzeri FM (2011) Effect of bout duration on exercise intensity and technical performance of small-sided games in soccer. $\mathbf{J}$ Strength Cond Res 25(2):453-458. https://doi.org/10.1519/JSC. 0b013e3181c1f8a2

13. Casamichana D, Castellano J, Dellal A (2013) Influence of different training regimes on physical and physiological demands during small-sided soccer games: Continuous vs. intermittent format. J Strength Cond Res. 27(3):690-697. https://doi.org/10. 1519/JSC.0b013e31825d99dc

14. Dellal A, Chamari K, Pintus A, Girard O, Cotte T, Keller D (2008) Heart rate responses during small-sided games and short intermittent running training in elite soccer players: a comparative study. J Strength Cond Res 22(5):1449-1457. https://doi. org/10.1519/JSC.0b013e31817398c6

15. Köklü Y, Sert Ö, Alemdaroğlu U, Arslan Y (2015) Comparison of the physiological responses and time-motion characteristics of young soccer players in small-sided games: the effect of goalkeeper. J Strength Cond Res 29(4):964-971. https://doi.org/10. 1519/JSC.0b013e3182a744a1

16. Hulka K, Weisser R, Belka J (2016) Effect of the pitch size and presence of goalkeepers on the work load of players during small-sided soccer games. J Hum Kinet 50(2):175-181. https:// doi.org/10.1515/hukin-2015-0180

17. Fernández-Espínola C, Robles MTA, Fuentes-Guerra FJG (2020) Small-sided games as a methodological resource for team sports teaching: a systematic review. IJERPH 17(6):1884. https://doi.org/10.3390/ijerph17061884

18. Bujalance-Moreno P, Latorre-Román PÁ, García-Pinillos F (2019) A systematic review on small-sided games in football players: acute and chronic adaptations. J Sports Sci 37(8):921949. https://doi.org/10.1080/02640414.2018.1535821

19. Hodgson C, Akenhead R, Thomas K (2014) Time-motion analysis of acceleration demands of $4 \mathrm{v} 4$ small-sided soccer games played on different pitch sizes. Hum Mov Sci 33(1):25-32. https://doi.org/10.1016/j.humov.2013.12.002

20. Kelly DM, Drust B (2009) The effect of pitch dimensions on heart rate responses and technical demands of small-sided soccer games in elite players. J Sci Med Sport 12(4):475-479. https://doi.org/10.1016/j.jsams.2008.01.010

21. Casamichana D, Castellano J (2010) Time-motion, heart rate, perceptual and motor behaviour demands in small-sides soccer 
games: effects of pitch size. J Sports Sci 28(14):1615-1623. https://doi.org/10.1080/02640414.2010.521168

22. Köklü Y, Albayrak M, Keysan H, Alemdaroğlu U, Dellal A (2013) Improvement of the physical conditioning of young soccer players by playing small-sided games on different pitch size-special reference to physiological responses. Kinesiology 45(1):41-47

23. Borg G (1998) Borg's perceived exertion and pain scales. Human kinetics, Champaign, IL

24. Beato M, Devereux G, Stiff A (2018) Validity and reliability of global positioning system units (STATSports Viper) for measuring distance and peak speed in sports. J Strength Cond Res 32(10):2831-2837. https://doi.org/10.1519/JSC.0000000000 002778

25. Duffield R, Reid M, Baker J, Spratford W (2010) Accuracy and reliability of GPS devices for measurement of movement patterns in confined spaces for court-based sports. J Sci Med Sport 13(5):523-525. https://doi.org/10.1016/j.jsams.2009.07.003

26. Carling C, Gall FL, Reilly TP (2010) Effects of physical efforts on injury in elite soccer. Int J Sports Med 31(03):180-185. https:// doi.org/10.1055/s-0029-1241212

27. Edwards S (1993) High performance training and racing. Hear rate monitor book, 8th edition: 113-123.

28. Impellizzeri FM, Rampinini E, Coutts AJ, Sassi A, Marcora SM (2004) Use of RPE-based training load in soccer. Med Sci Sports Exerc 36(6):1042-1047. https://doi.org/10.1249/01.MSS.00001 28199.23901.2F
29. Fanchini M, Ferraresi I, Modena R, Schena F, Coutts AJ, Impellizzeri FM (2016) Use of the CR100 scale for session rating of perceived exertion in soccer and its interchangeability with the CR10. Int J Sports Physiol Perform 11(3):388-392. https://doi. org/10.1123/ijspp.2015-0273

30. Impellizzeri FM, Marcora SM, Castagna C et al (2006) Physiological and performance effects of generic versus specific aerobic training in soccer players. Int J Sports Med 27(6):483-492. https:// doi.org/10.1055/s-2005-865839

31. Borg E, Borg G (2002) A comparison of AME and CR100 for scaling perceived exertion. Acta Psychol (Amst). 109(2):157-175. http://www.ncbi.nlm.nih.gov/pubmed/11820425. Accessed 22 Sep 2018.

32. Lakens D (2013) Calculating and reporting effect sizes to facilitate cumulative science: a practical primer for t-tests and ANOVAs. Front Psychol. 4:863. https://doi.org/10.3389/fpsyg.2013.00863

33. McLaren SJ, Smith A, Spears IR, Weston M (2017) A detailed quantification of differential ratings of perceived exertion during team-sport training. J Sci Med Sport 20(3):290-295. https://doi. org/10.1016/j.jsams.2016.06.011

Publisher's Note Springer Nature remains neutral with regard to jurisdictional claims in published maps and institutional affiliations. 